Orthopäde 2017 · 46:1-3

DOI 10.1007/s00132-016-3353-7

Online publiziert: 15 . November 2016

(c) Springer Medizin Verlag Berlin 2016

CrossMark

\section{Jäger}

Klinik für Orthopädie und Unfallchirurgie, Universität Duisburg-Essen, Essen, Deutschland

\title{
Was uns ältere Patienten wert sind
}

Aufgrund der besonderen demographischen Entwicklung in Deutschland hat die medizinische Versorgung des älteren Menschen inzwischen gesundheitspolitisch einen sehr hohen Stellenwert erlangt. Berücksichtigt man die gesetzgeberischen Vorgaben, wie u. a. anhand der Landesbettenplanungen der Länder mit Ausbau geriatrisch-psychiatrischer Einrichtungen aufgezeigt, darf man davon ausgehen, dass sich dieser Trend auch in den nächsten Jahren fortsetzen wird. Dementsprechend groß sind die Herausforderungen auf orthopädischunfallchirurgischem Fachgebiet. Neben dem Ausbau der Alterstraumatologie und der Behandlung degenerativer Erkrankungen der Wirbelsäule rückt hier insbesondere der Gelenkersatz in den Fokus der Fachwelt und Öffentlichkeit. So wurden im vergangenen Jahr in Deutschland über 340.000 Hüft- und Kniegelenke endoprothetisch ersetzt, davon der überwiegende Anteil bei älteren Menschen. Im aktuellen „Weißbuch Gelenkersatz" wird näher auf die besondere nationale Versorgungssituation endoprothetischer Hüft- und Knieoperationen eingegangen.

Wesentliche Aspekte in der Endoprothetik beim älteren Menschen betreffen die Patientensicherheit, handelt es sich bei diesem besonderen Kollektiv doch um Individuen mit speziellen Risikoprofilen. So ist der stationäre Aufenthalt des geriatrischen Patienten häufig aufgrund von Begleiterkrankungen und besonderen sozialen Herausforderung komplex und in zahlreichen Fällen auch problembehaftet. Um dieser medizinischen Herausforderung angemessen entgegenzutreten, müssen besondere infrastrukturelle, aber auch logistische Strukturen in den jeweiligen klinischen
Zentren vorgehalten werden. Hierzu gehört eine gut abgestimmte interdisziplinäre Vorbereitung mit frühzeitiger konsilarischer Einbindung der Anästhesiologie. Im Falle von Belastungsdyspnoe oder Stenokardie sowie bei bekannten Vorerkrankungen ist ebenfalls die Indikation zur kardiologischen Abklärung präoperativ großzügig zu stellen.

Darüber hinaus muss für ein rechtzeitiges mikrobiologisches Screening und ggf. eine medikamentöse Umstellung im Falle einer vorbestehenden Antikoagulation („bridging“) im Vorfeld der Operation Sorge getragen werden. Hierbei ist ebenso die enge Kommunikation mit dem niedergelassenen Arzt erforderlich. Bereits vor der stationären Aufnahme muss die häusliche soziale Situation und die poststationäre Unterbringung des $\mathrm{Pa}$ tienten geklärt sein. Hierbei sind regelmäßig die Angehörigen und der Sozialdienst einzubeziehen.

\section{I) Eine gute Patientenführung und altersgerechte Rehabilitation ist wichtig}

Insbesondere die unmittelbare perioperative Antikoagulation erfordert im Sinne einer kritischen Risiko-Nutzen-Abwägung bei kardiologischen Risikopatienten höchst individuelle Entscheidungen. Hierbei kommt mitunter der verantwortliche Operateur zu einem anderen Ergebnis wie dessen internistische Kollegen. Enge Absprachen und eine gute Dokumentation sind hier erforderlich. Aber auch bei der Indikationsstellung zur Gabe von Blutprodukten („blood management") stoßen die einschlägigen Leitlinien auf diesem Gebiet an ihre Grenzen. Diese beziehen sich in der Regel auf an- dere Patientenkollektive (z. B. Polytraumatisierte) und lassen sich nicht auf die Endoprothetik beim älteren Menschen übertragen.

Es ist sehr erfreulich, dass moderne Implantatsysteme, sowohl bei zementierter als auch zementfreier Verankerung, eine Frühmobilisierung unserer älteren Patienten erlauben. Jedoch kann nur der Operateur die lokale Knochenqualität und damit das Ausmaß der postoperativen Belastung einschätzen. Mehrwöchige vorausgegangene Bettlägerigkeit, kardiovaskuläre und pulmonale Vorerkrankungen, eine Demenz sowie ein insuffizienter Ernährungs- und Muskelstatus erschweren darüber hinaus die Mobilisation. Gerade ältere Patienten jenseits des 80 . Lebensjahres benötigen pflegerischer- als auch physiotherapeutischerseits eine intensive Zuwendung und Betreuung.

Die klinische Erfahrung zeigt jedoch, dass unter dem Druck einer auf Basis des Instituts für das Entgeltsystem im Krankenhaus (InEK) basierten Kostenkalkulation dem Personal nicht die erforderliche Zeit zur Mobilisation dieser Patienten zur Verfügung steht. Daherverwundert es auch nicht, dass die aus dem marktwirtschaftlichen Bereich entlehnte Begriffe wie "fast track surgery" oder ,rapid recovery" für diese Patienten medizinisch nicht sinnvoll umsetzbar sind, wie aktuelle Studien aus der wissenschaftlichen Literatur belegen. Es wird prognostiziert, dass eine bereits unterfinanzierte Versorgung dieses Patientenkollektivs unter der aktuellen Abwertung der DRG zu einer weiteren Verschärfung des Problems beitragen werden.

Die älteren Patienten, welche einen Gelenkersatz benötigen, bilden jedoch keineswegs eine homogene Gruppe. Ne- 
ben den multimorbiden haben wir es auch mit ,junggebliebenen “ Patienten zu tun, die durchaus neben dem Ziel einer schmerzfreien Mobilität auch im fortgeschrittenen Alter sportliche Ambitionen verfolgen. Hierunter finden sich auch $\mathrm{Pa}$ tienten jenseits des 80. Lebensjahrs. Zwar ist bei diesen Patienten in der Regel ein hohes Maß an Compliance gegeben. Eine gute Patientenführung und altersgerechte Rehabilitation mit stufenweiser Wiedereingliederung in den Alltag ist gerade bei diesen nicht selten überehrgeizigen Patienten wichtig, um diese vor einer Überlastung zu schützen. Einschlägiges Informationsmaterial und eine gute präoperative Vorbereitung kann hierbei die Patientenführung ergänzen, diese jedoch nicht ersetzen.

Eine weitere Gruppierung älterer Patienten mit endoprothetischem Ersatz fällt in den Bereich der Alterstraumatologie. Meist liegen Bagatelltraumen zugrunde, die aufgrund einer insuffizienten Knochenqualität zur Fraktur führen. Ebenso ist der Anteil kardiologisch vorerkrankter und dementer Patienten hoch. Besonders für dieses Kollektiv ist daher eine zeitnahe Versorgung geboten. Die Kliniken müssen sicherstellen, dass rund um die Uhr einschlägig ausgebildete und auf dem Gebiet der Endoprothetik erfahrene Operateure vor Ort sind. Anzustreben sind hierbei kurze Operationszeiten und Erfahrung im Umgang mit lokalen Knochendefekten und minderer Knochenqualität, mit pathologischen Frakturen sowie mit hüftgelenknahen Deformitäten. Zur Sicherstellung einer ausreichenden Behandlungsqualität muss die operative Erfahrung bei hüftgelenknahen Frakturen des älteren Menschen daher die einer Duokopfimplantation bei weitem übersteigen.

All die angesprochenen Aspekte verdeutlichen, dass die Endoprothetik beim älteren Menschen deutlich aufwendiger ist, als dies die reine operative Leistung "Gelenkersatz" zunächst suggeriert. Bei all der Forderung nach einem hohen Maß an Standardisierungen in den Behandlungsabläufen, wie sie sinnvollerweise von den Zertifizierungsgremien großer Endoprothetikzentren propagiert werden, erfordert die Altersendoprothetik am Ende doch eine sehr individuelle
Patientenführung. Letztendlich ist und bleibt sie damit eine Sonderform der personalisierten Medizin. Dieser Aufgabe kann jedoch nur beim Vorliegen entsprechender personeller sowie ökonomischer Ressourcen gerecht werden.

In diesem Kontext geraten die behandelnden Ärzte seit einigen Jahren immer stärker in das Spannungsfeld gesetzlicher und behördlicher Vorgaben sowie ökonomischer Forderungen durch die Krankenhaus- und Kostenträger. In kaum einem anderen Bereich der operativen Medizin greift die Ökonomisierung der Medizin so weitreichend um sich wie in der Endoprothetik. Unsere wissenschaftlichen Fachgesellschaften versuchen, durch das Erstellen von Leitlinien und die Auswertung wissenschaftlich fundierten Daten aus der Versorgungsforschung diese Entwicklungen zu begleiten.

Aber auch weitab des Operationssaales und der Medizin am Krankenbett werden von übergeordneter Stelle Entscheidungsvorlagen erarbeitet und den entsprechenden Gremien zur Abstimmung vorgelegt. Hierbei besteht die besondere Gefahr, dass die aktiven und operativ tätigen Fachvertreter nicht ausreichend Gehör finden und eine Entkoppelung zwischen Entscheidung und Verantwortung stattfindet. Dem Institut für Qualitätssicherung und Transparenz im Gesundheitswesen (IQTIG) sowie dem gemeinsamen Bundesausschuss (G-BA) kommt hierbei eine Schlüsselrolle und damit eine besondere Verantwortung zu. Als Beispiele für die skizzierte Entwicklung werden die anhaltende Diskussion um Mindestmengen in der Endoprothetik als Grundlage für eine Vergütung, das 24Stunden-Intervall zur Versorgung hüftgelenknaher Femurfrakturen oder das Infragestellen jahrzehntelang bewährter Medizinprodukte aus vermeintlichen Sterilitätsgründen angeführt. So droht die Schere zwischen den Forderungen einerseits und der klinischen Umsetzbarkeit andererseits auf dem Gebiet der Endoprothetik immer weiter auseinanderzuklaffen. Hiervon ist insbesondere der ältere Mensch mit einer hochindividuellen Ausgangssituation betroffen.
Am Ende des Tages muss die Gesellschaft selbst entscheiden, inwieweit sie bereit ist, Hochleistungsimplantate für unsere betagten Mitbürgerinnen und Mitbürger mit begrenzter Lebenserwartung $\mathrm{zu}$ finanzieren, und ob und inwieweit der medizinisch indizierte Gelenkersatz ökonomischen Zielen geopfert werden darf.

Als Mentoren für unsere Patienten haben Orthopäden und Unfallchirurgen in Deutschland die besondere Verantwortung, sich an dieser Diskussion aktiv zu beteiligen und wissenschaftlich fundierte Daten als Entscheidungshilfen den Entscheidungsträgern zur Verfügung zu stellen.

Die nachfolgenden Beiträge greifen wesentliche Aspekte der Altersendoprothetik auf und diskutieren diese vor dem Hintergrund wissenschaftlich gesicherter Daten.

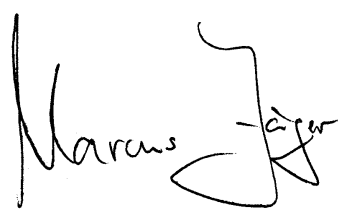

Marcus Jäger

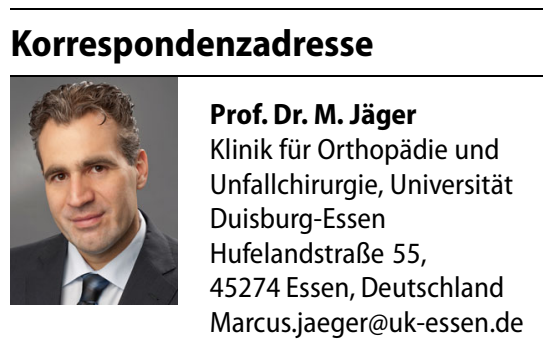

Interessenkonflikt. M. Jäger gibt an, dass kein Interessenkonflikt besteht. 
Hier steht eine Anzeige.

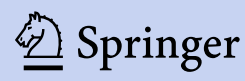

